\section{Cureus}

Received 02/15/2017

Review began 03/13/2017

Review ended 03/13/2017

Published 03/21/2017

\section{C) Copyright 2017}

Mangi et al. This is an open access article distributed under the terms of the Creative Commons Attribution License CC-BY 3.0., which permits unrestricted use, distribution, and reproduction in any medium, provided the original author and source are credited.

\title{
Association of Non-alcoholic Fatty Liver Disease with Conduction Defects on Electrocardiogram
}

\author{
Muhammad A. Mangi ${ }^{1}$, Abdul M. Minhas ${ }^{2}$, Hiba Rehman ${ }^{1}$, Furquan Pathan ${ }^{1}$, Hong Liang ${ }^{3}$, \\ Sary Beidas 1 \\ 1. GME Internal Medicine, Orange Park Medical Center 2. Internal Medicine, Orange Park Medical Center \\ 3. GME Internal Medicine Residency Program, North Florida Regional Medical Center
}

$\square$ Corresponding author: Muhammad A. Mangi,drmasif33@yahoo.com

Disclosures can be found in Additional Information at the end of the article

\section{Abstract}

Background: Non-alcoholic fatty liver disease (NAFLD) is a leading cause of liver disease in developed countries. The association of NAFLD with conduction defects is unknown. The aim of our study was to find whether an association exists between conduction defects and NAFLD.

Methods: This is a case-control retrospective study of 700 patients admitted to Orange Park Medical Center, Orange Park, Florida from 2009 to 2015. Patients with a history of alcohol use, congenital heart disease, infiltrative malignancy, and myocarditis were excluded from the study. NAFLD was diagnosed by detection of hepatic steatosis on abdominal ultrasound or computerized tomography (CT) scan. Electrocardiograms (EKGs) were performed on all 700 patients and were interpreted by a cardiologist. Univariate logistic regression was used to assess the association between NAFLD and the variables of demographics, clinical characteristics, medicine use, EKG changes, and conduction defects, while multivariate logistic regression with backward elimination method was performed to determine if NAFLD is one of the most important risk factors for conduction defects.

Results: The study population included 408 patients with NAFLD and 292 patients with NoNAFLD. A total of 155 conduction defects occurred in 140 patients; conduction defects included 25.7\% (36) patients with first degree block, 2.1\% (three) patients with Mobitz type 1 block, 41.4\% (58) patients with right bundle branch block (RBBB), 17.9\% (25) patients with left bundle branch block (LBBB), 11.4\% (16) patients with bifascicular block, and 12.1\% (17) patients with nonspecific intraventricular block. Multivariate logistic regression with backward elimination method identified six risk factors for conduction defects; these included NAFLD (odds ratio (OR) 2.38; 95\% confidence interval (CI) 1.51-3.73, p<0.0001), hypertrophy (OR 2.52; 95\% CI 1.57-4.05, $\mathrm{p}=0.0001)$, congestive heart failure (CHF) (OR 3.05; 95\%CI 1.46-6.38, $\mathrm{p}=0.0031)$, male sex (OR 1.79; 95\%CI 1.19-2.69, p=0.0051), diabetes mellitus (OR 1.63; 95\% CI 1.08-2.47, $\mathrm{p}=0.02$ ), and age (OR 1.04; 95\% CI 1.02-1.06, $\mathrm{p}<0.0001$ ).

Conclusion: NAFLD is associated with conduction defects. Prospective randomized trials are needed to demonstrate that NAFLD causes conduction defects.

Categories: Cardiology, Internal Medicine, Gastroenterology Keywords: nafld, conduction defects, ekg changes 


\section{Introduction}

Non-alcoholic fatty liver disease (NAFLD) is defined as fatty infiltration of the liver in the absence of alcohol use or infection with viral hepatitis. NAFLD is a common disorder, affecting $25 \%$ of the world population and almost $24 \%-32 \%$ of the population in the western world [1]. In the USA, NAFLD affects $30 \%$ of the population and is the third most common indication for liver transplant [2-3]. This high prevalence of NAFLD is presumably caused by a high-calorie diet and a sedentary lifestyle [4]. In fact, NAFLD is projected to become the most common cause of end-stage liver disease and liver transplant in the next decade [5-6]. Furthermore, evidence suggests that NAFLD is a multi-system disease that affects the liver and the cardiovascular system leading to structural and functional changes in the heart and the blood vessels. Ultimately these changes are responsible for the increased cardiac morbidity and mortality associated with NAFLD [7-9].

To date, there has been one retrospective study to ascertain the association of NAFLD with conduction defects [10]. The study demonstrated that patients with right bundle branch block (RBBB) are at higher risk of developing NAFLD due to passive congestion of the liver. We extend this line of thought and postulate that patients with NAFLD are at risk for developing other types of conduction defects. In order to test this hypothesis, we conducted a large populationbased case-control study of adult men and women at Orange Park Medical Center (OPMC) in Florida.

\section{Materials And Methods}

The study protocol was approved by the OPMC Graduate Medical Education Research committee as a minimal risk study because the study is a retrospective chart review with no patient contact.

\section{Study design}

This was a retrospective case-control study designed to test the association between NAFLD and conduction defects (Figure 1). NAFLD was an incidental finding in patients admitted for other reasons. NAFLD was diagnosed by detection of hepatic steatosis on abdominal ultrasonography (USG) or computerized tomography (CT) scans. Patients were divided into two groups: group-1 included patients who had NAFLD diagnosed on abdominal imaging and group-2 were controls who did not have NAFLD on abdominal imaging. Controls (No-NAFLD) were randomly selected from the general population of hospitalized adult patients, who also had an electrocardiogram (EKG) and abdominal imaging studies performed during hospitalization. All EKGs were interpreted by a cardiologist independent of the study.

\section{Study population}

The study population included adult patients ( $>18$ years of age) admitted to the OPMC from 2009 to 2015. All patients had abdominal imaging and EKG studies ordered. Based on abdominal imaging, the population was divided into NAFLD versus No-NAFLD cases. Patients were excluded if there was a history of alcohol use, defined as $>21$ drinks/week for men and $>14$ drinks/week for women. Other exclusions included acute viral hepatitis, chronic viral hepatitis, congenital heart disease, infiltrative malignancy, myocarditis or cardiac surgeries. Also, patients who did not have an abdominal USG, CT scan abdomen or EKG were excluded from the study.

\section{Predictor variables}

Baseline demographic characteristics collected included age, gender, race, obese, chronic conditions (asthma/chronic obstructive pulmonary disease (COPD), congestive heart failure 
(CHF), diabetes mellitus, hypertension) ischemic heart disease, NAFLD, medication use (betablockers, calcium channel blockers, digoxin, amiodarone, adenosine), cirrhosis, thyroid disorders, smoking, cocaine use (Table 1). We did not include body mass index (BMI) in our study due to lack of availability of height in the medical record for most patients.

\section{Outcome variables}

Conduction defect identified on EKG was the primary outcome variable. The EKG changes for conduction defects were determined by reviewing EKG tracings already verified by a cardiologist. Secondary outcomes for this study included the presence of other EKG changes (for example, atrial fibrillation, premature atrial contractions (PACs), premature ventricular contractions (PVCs), axis deviation, low voltage, prolonged QTc interval, hypertrophy, and ST wave changes).

\section{Statistical analysis}

Univariate logistic regression analysis was used to assess the association between NAFLD, baseline demographics, clinical characteristics, medicine use, EKG changes, and conduction defects (Table 2). Multivariate logistic regression analysis with backward elimination method was performed to determine if NAFLD is a risk factor for conduction defects from 19 selected predictors/factors (age, sex, race, obesity, coronary artery disease (CAD), hypertension, diabetes mellitus, CHF, smoking, COPD, asthma, thyroid disorder, antipsychotic medicine, hyperlipidemia, and NAFLD) (Table 3). The risk estimates were reported as odds ratios (OR) with $95 \%$ confidence intervals (CI). A p-value $<0.05$ was considered statistically significant. $\mathrm{R}$ version 3.3.1 (University of Auckland, New Zealand) was primarily used for statistical analysis and SAS 9.4 (SAS Institute Inc., Cary, NC) was used to validate R output. 


\section{Cureus}

\section{Study Design Flowchart}

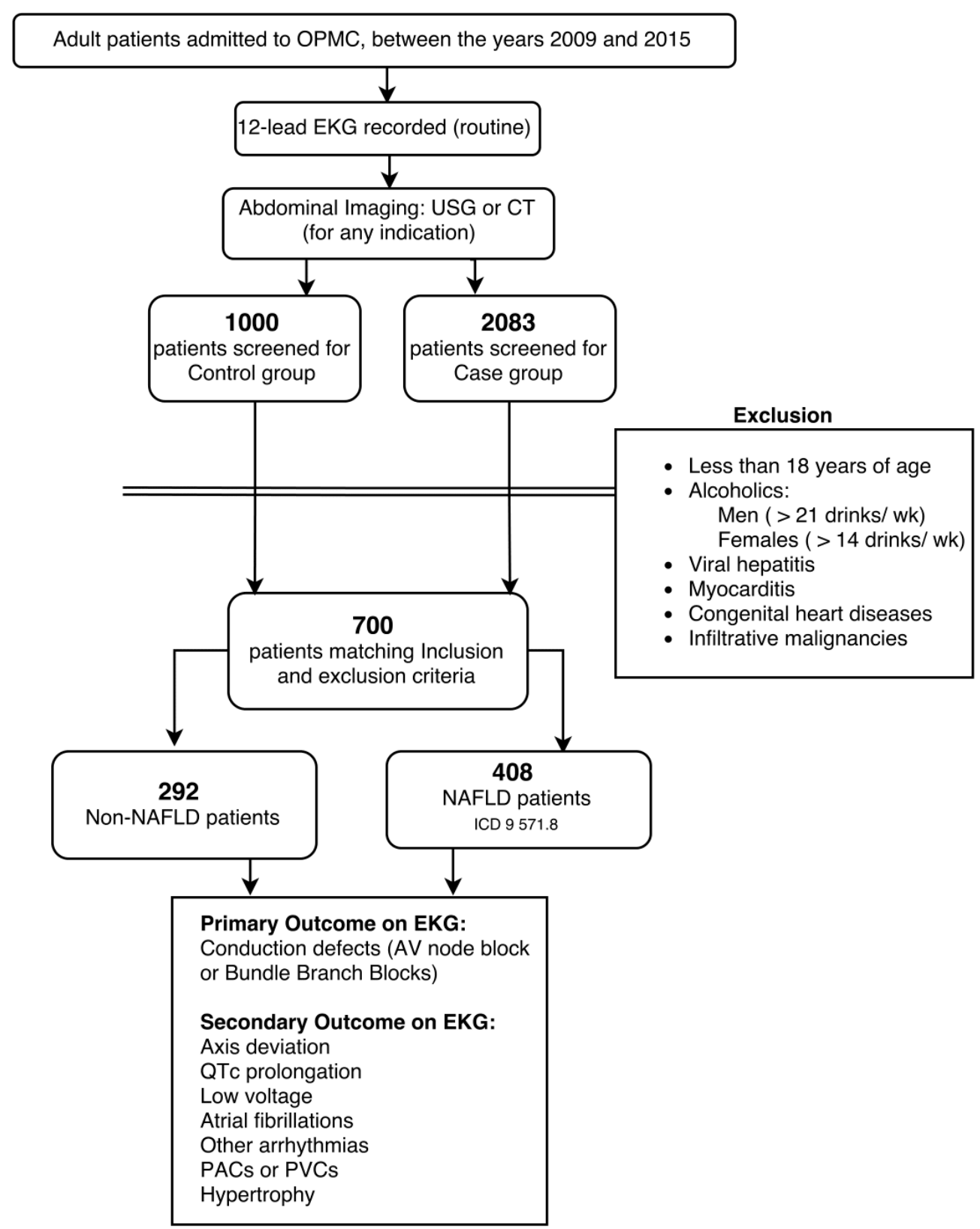

FIGURE 1: Study design flowchart

\section{Results}

A total of 700 patients were included in the study. NAFLD was identified on abdominal imaging in 408 patients. In the No-NAFLD group, there were 292 patients. The median age was 58 years (standard deviation $(\mathrm{SD})=15.3$ years). There were 293 males (41.9\%), and 407 females (58.1\%). Most patients were Caucasian $(n=534,76.3 \%)$. Other baseline characteristics are listed below (Table 1). A total of 155 conduction defects were identified in 140 patients demonstrated on EKG (Table 4). 


\section{Cureus}

\begin{tabular}{|c|c|c|c|c|c|c|}
\hline \multirow[b]{3}{*}{ Age (median)(SD) } & \multicolumn{2}{|l|}{ ALL } & \multicolumn{2}{|c|}{ NAFLD } & \multicolumn{2}{|c|}{ No NAFLD } \\
\hline & \multicolumn{2}{|c|}{$\mathrm{N}, \%$ or Median, SD } & \multicolumn{2}{|c|}{$\mathrm{N}, \%$ or Median, SD } & \multicolumn{2}{|c|}{$\mathrm{N}, \%$ or Median, SD } \\
\hline & 57.9 & (15.3) & 59 & $(13.6)$ & 56.3 & (17.5) \\
\hline Sex (males) & 293 & (41.8\%) & 178 & (43.6\%) & 115 & (39.4\%) \\
\hline (females) & 307 & $(58.2 \%)$ & 230 & $(56.4 \%)$ & 177 & $(60.8 \%)$ \\
\hline \multicolumn{7}{|l|}{ Race } \\
\hline Caucasian & 534 & (76.4\%) & 318 & $(80 \%)$ & 216 & $(74.2 \%)$ \\
\hline African American & 98 & $(14 \%)$ & 45 & (11\%) & 53 & $(18.3 \%)$ \\
\hline Hispanic & 17 & (0.02\%) & 16 & (3.9\%) & 1 & $(0.3 \%)$ \\
\hline Other & 50 & (0.07\%) & 29 & (7.1\%) & 21 & (7.2\%) \\
\hline Smokers & 237 & (33.8\%) & 142 & $(34.8 \%)$ & 96 & $(32.9 \%)$ \\
\hline |llicit Drug Users & 73 & (10.4\%) & 46 & (11.3\%) & 27 & $(9.3 \%)$ \\
\hline Hypertensive & 421 & (60.1\%) & 262 & $(64.2 \%)$ & 159 & $(54.5 \%)$ \\
\hline CAD & 94 & $(13.4 \%)$ & 45 & $(11.0 \%)$ & 49 & $(16.8 \%)$ \\
\hline Hyperlipidemia & 230 & (32.8\%) & 123 & $(30.2 \%)$ & 107 & $(36.6 \%)$ \\
\hline Diabetics & 224 & (32\%) & 156 & $(38.2 \%)$ & 68 & $(23.3 \%)$ \\
\hline Obese & 339 & (48.4\%) & 214 & $(52.5 \%)$ & 125 & $(42.8 \%)$ \\
\hline CHF & 38 & $(0.05 \%)$ & 23 & $(5.6 \%)$ & 15 & $(5.1 \%)$ \\
\hline
\end{tabular}

TABLE 1: Demographics and clinical characteristics of the study population

\section{Univariate analysis}

Univariate analysis of EKG findings, (conduction defect, PACs/PVCs, hypertrophy, axis deviation, ST wave changes, low voltage, and QTc prolongation) identified a positive association with NAFLD ( $\mathrm{p}$-value $<0.05$ ). Atrial fibrillation showed no association with NAFLD (Table 2).

The proportion of patients with a conduction defect on EKG was higher in patients with NAFLD $(107 / 408,26.2 \%)$ compared to the No-NAFLD group (33/292, 11.3\%), (p-value $<0.001)$. When conduction defects were further stratified (Table 4) into AV block and bundle branch block, AV block was not significantly associated with NAFLD (6.9\% in NAFLD vs $3.8 \%$ No-NAFLD (p-value $0.0828)$. However, bundle branch block was significantly associated with NAFLD $(22.1 \%$ in NAFLD vs $8.9 \%$ in No-NAFLD (p-value $<0.001$ ). 


\section{Cureus}

\begin{tabular}{|c|c|c|c|c|c|c|}
\hline \multirow[b]{2}{*}{ Mean age, year (SD) } & \multicolumn{2}{|c|}{$\mathrm{N}, \%$ or Mean, SD } & \multicolumn{2}{|c|}{$\mathrm{N}, \%$ or Mean, SD } & \multirow[b]{2}{*}{$1.01(1.00-1.02)$} & \multirow{2}{*}{0.0203} \\
\hline & 59.0 & (13.6) & 56.3 & (17.5) & & \\
\hline Sex (male) & 178 & (43.6) & 115 & (39.4) & $1.19(0.88-1.62)$ & 0.2619 \\
\hline Race (African American) & 45 & (11.0) & 53 & (18.2) & $0.56(0.36-0.86)$ & 0.0080 \\
\hline Smokers (current/former) & 142 & $(34.8)$ & 96 & (32.9) & $1.09(0.79-1.50)$ & 0.5956 \\
\hline Hypertensive & 262 & (64.2) & 159 & (54.5) & $1.50(1.11-2.04)$ & 0.0094 \\
\hline CAD & 45 & (11.0) & 49 & (16.8) & $0.62(0.40-0.95)$ & 0.0288 \\
\hline Hyperlipidemia & 123 & $(30.2)$ & 107 & (36.6) & $0.75(0.54-1.03)$ & 0.0715 \\
\hline Diabetics Mellitus & 156 & (38.2) & 68 & (23.3) & $2.04(1.46-2.86)$ & $<0.0001$ \\
\hline Obese & 214 & (52.5) & 125 & (42.8) & $1.47(1.09-1.99)$ & 0.0120 \\
\hline $\mathrm{CHF}$ & 23 & (5.6) & 15 & (5.1) & $1.11(0.57-2.16)$ & 0.7676 \\
\hline Cirrhosis & 73 & (17.9) & 2 & (0.68) & 31.59 (7.69-129.84) & $<0.0001$ \\
\hline COPD & 59 & (14.5) & 34 & (11.6) & $1.28(0.82-2.02)$ & 0.2798 \\
\hline Asthma & 12 & (2.9) & 14 & (4.8) & $0.60(0.27-1.32)$ & 0.2054 \\
\hline PAC/PVC & 86 & (21.1) & 29 & (9.9) & $2.42(1.54-3.80)$ & 0.0337 \\
\hline Thyroid disorder & 78 & (19.1) & 41 & (14.0) & $1.45(0.96-2.19)$ & 0.0789 \\
\hline Mllicit Drug Users & 46 & (11.3) & 27 & $(9.3)$ & $1.25(0.76-2.06)$ & 0.3874 \\
\hline Beta Blocker & 130 & (31.9) & 92 & (31.5) & $1.02(0.74-1.40)$ & 0.9206 \\
\hline Digoxin & 4 & (1.0) & 1 & $(0.3)$ & $2.88(0.32-25.91)$ & 0.3450 \\
\hline TCA & 13 & (3.2) & 5 & (1.7) & $1.89(0.67-5.36)$ & 0.2321 \\
\hline SSRI & 75 & (18.4) & 37 & (12.7) & $1.55(1.01-2.38)$ & 0.0432 \\
\hline CCB & 80 & (19.6) & 59 & $(20.2)$ & $0.96(0.66-1.40)$ & 0.8448 \\
\hline Antipsychotic & 21 & (5.2) & 14 & (4.8) & $1.08(0.54-2.16)$ & 0.8339 \\
\hline Amiodarone & 0 & $(0.0)$ & 5 & (1.7) & $<0.001 \quad(<0.001->999.9)$ & 0.9797 \\
\hline Adenosine & 0 & $(0.0)$ & 0 & $(0.0)$ & NA & NA \\
\hline Atrial fibrillation & 45 & (11.0) & 33 & (11.3) & $0.97(0.60-1.57)$ & 0.9101 \\
\hline Low Voltage & 57 & 14.0) & 17 & (5.8) & $2.63(1.49-4.62)$ & 0.0008 \\
\hline QT-prolongation & 93 & (22.8) & 16 & (5.5) & $5.09(2.92-8.86)$ & $<0.0001$ \\
\hline ST-change & 195 & (47.8) & 115 & (39.4) & $1.41(1.04-1.91)$ & 0.0274 \\
\hline Anterioseptal-ST & 49 & (12.0) & 33 & (11.3) & $1.07(0.67-1.71)$ & 0.7749 \\
\hline Anteriolateral-ST & 45 & (11.0) & 30 & (10.3) & $1.08(0.66-1.76)$ & 0.7514 \\
\hline
\end{tabular}




\section{Cureus}

\begin{tabular}{|lllllll}
$\quad$ Interior-ST & 33 & $(8.1)$ & 20 & $(6.9)$ & $1.20(0.67-2.13)$ & 0.5417 \\
$\quad$ Posterior-ST & 3 & $(0.7)$ & 0 & $(0.0)$ & $>999(<0.01->999.9)$ & 0.9845 \\
$\quad$ Nonspecific-ST & 124 & $30.4)$ & 50 & $(17.1)$ & $2.11(1.45-3.06)$ & $<0.0001$ \\
Axis deviation & 91 & $(22.3)$ & 24 & $(8.2)$ & $3.21(1.99-5.17)$ & $<0.0001$ \\
Hypertrophy & 91 & $(22.3)$ & 33 & $(11.3)$ & $2.25(1.46-3.47)$ & 0.0002 \\
Conduction Defect & 107 & $(26.2)$ & 33 & $(11.3)$ & $2.79(1.83-4.26)$ & $<0.0001$ \\
$\quad$ AV block & 28 & $(6.9)$ & 11 & $(3.8)$ & $1.88(0.92-3.84)$ & 0.0828 \\
$\quad$ Bundle Branch Block & 90 & $(22.1)$ & 26 & $(8.9)$ & $2.90(1.82-4.61)$ & $<0.0001$
\end{tabular}

TABLE 2: Univariate analysis of demographics, clinical characteristics, medicine, and EKG changes by NAFLD

TCA - tricyclic antidepressant, SSRI - selective serotonin reuptake inhibitors, CCB - calcium channel blocker.

\section{Multivariate analysis}

Multivariate logistic regression with backward elimination identified six risk factors for conduction defects including; NAFLD (OR 2.38; 95\% CI 1.51-3.73, p <0.0001), hypertrophy (OR 2.52; 95\% CI 1.57-4.05, p=0.0001), CHF (OR 3.05; 95\% CI 1.46-6.38, p=0.0031), male sex (OR 1.79; 95\% CI 1.19-2.69, p=0.0051), diabetes mellitus (OR 1.63; 95\% CI 1.08-2.47, p=0.02), and age (OR 1.04; 95\% CI 1.02-1.06, p<0.0001) (Table 3).

\begin{tabular}{|c|c|c|c|c|c|c|}
\hline \multirow[t]{2}{*}{ Variables\# } & \multicolumn{2}{|c|}{ Conduction Defect (140) } & \multicolumn{2}{|c|}{ No-Conduction Defect (560) } & Odds ratio $(95 \% \mathrm{Cl})$ & p-value \\
\hline & \multicolumn{2}{|c|}{$\mathrm{N}, \%$ or Mean, SD } & \multicolumn{2}{|c|}{$\mathrm{N}, \%$ or Mean, SD } & \multirow[b]{2}{*}{$1.04(1.02-1.06)$} & \multirow[b]{2}{*}{$<0.0001$} \\
\hline Mean age, year (SD) & 64.5 & (14.3) & 56.3 & $(15.3)$ & & \\
\hline Sex (male) & 71 & $(50.7)$ & 222 & $(39.6)$ & 1.79 (1.19-2.69) & 0.0051 \\
\hline Diabetics Mellitus & 62 & $(44.3)$ & 162 & $(28.9)$ & $1.63(1.08-2.47)$ & 0.0205 \\
\hline $\mathrm{CHF}$ & 17 & $(12.1)$ & 21 & $(3.8)$ & $3.05(1.46-6.38)$ & 0.0031 \\
\hline Hypertrophy & 42 & $(30.0)$ & 82 & $(14.6)$ & $2.52(1.57-4.05)$ & 0.0001 \\
\hline NAFLD & 107 & $(76.4)$ & 301 & $(53.8)$ & $2.38(1.51-3.73)$ & $<0.0001$ \\
\hline
\end{tabular}

TABLE 3: Multivariate logistic regression analysis for conduction defect using backward elimination method 


\section{Cureus}

\begin{tabular}{|c|c|c|c|c|c|c|}
\hline \multirow[t]{2}{*}{ Variables } & \multicolumn{2}{|c|}{ No-NAFLD (33) } & \multicolumn{2}{|c|}{ NAFLD (107) } & \multicolumn{2}{|c|}{ Total (140) } \\
\hline & $\mathrm{n}$ & $\%$ & $\mathrm{n}$ & $\%$ & $\mathrm{n}$ & $\%$ \\
\hline $1^{\text {st }}$ degree AV block & 11 & 7.1 & 25 & 16.1 & 36 & 23.2 \\
\hline Mobitz type 1 & 0 & 0.0 & 3 & 2.1 & 3 & 2.1 \\
\hline Right bundle branch block & 14 & 9.0 & 44 & 28.4 & 58 & 37.4 \\
\hline Left bundle branch block & 4 & 2.6 & 21 & 13.5 & 25 & 16.1 \\
\hline Bi-fascicular block & 1 & 0.6 & 15 & 9.7 & 16 & 10.3 \\
\hline Nonspecific intraventricular block & 7 & 4.5 & 10 & 6.5 & 17 & 11.0 \\
\hline Total conduction defects & 37 & 23.9 & 118 & 76.1 & 155 & 100.0 \\
\hline
\end{tabular}

TABLE 4: Conduction defect distribution

\section{Discussion}

The major finding of this study is that patients diagnosed with NAFLD showed a significant association with conduction defects. This association was independent of numerous other risk factors after adjusting for confounders.

Prior studies have shown associations between NAFLD and CAD [11-13]. However, few studies have evaluated the association of NAFLD with electrical abnormalities of the heart. To our knowledge, this is the first study to evaluate for the presence of conduction defects and describe EKG abnormalities found in patients with NAFLD.

The underlying pathophysiological mechanism responsible for the association between NAFLD and conduction defect is not well understood. NALFD might be a marker for ectopic fat deposition in myocardium and pericardium that promotes cardiovascular disease. A recent study has shown an association between intra-hepatic and myocardial triacylglycerol content and that increased pericardial fat is associated with increased prevalence of atrial fibrillation [14-15]. Further, adipocytes in retrosternal, epicardial tissue have been shown to exert effects on ion currents in rabbit left atria, leading to arrhythmias [16]. NAFLD may potentially contribute to the development of cardiovascular complications as demonstrated by the increased production of pro-inflammatory cytokines such as C-reactive protein, interleukin-6, tumor necrosis factor - alpha and prothrombotic factors [17-18]. These markers have been associated with structural changes in the heart and a higher rate of arrhythmias [19-21].

This study has several limitations. First, NAFLD was diagnosed by USG or CT scan, instead of tissue biopsy, the gold standard. Studies have shown that USG has a sensitivity of $60 \%$ to $94 \%$ and specificity of $84 \%$ to $95 \%$, while CT scan has a sensitivity of $82 \%$ and specificity of $100 \%$; so neither USG nor CT scan is able to reliably detect hepatic lipid content that is less than $30 \%$ [22-24]. Hence, we probably missed mild to moderate cases of NAFLD [24]. Furthermore, we were unable to differentiate NAFLD from non-alcoholic steatohepatitis, as no tissue biopsy results were available. Second, patients were not primarily admitted for NAFLD, rather NAFLD was an incidental finding identified using International Classification of Diseases, Ninth Revision (ICD-9) codes. Finally, we cannot exclude the effect of unmeasured or unknown 
confounding factors. For instance, although NAFLD is strongly associated with obesity, we were unable to exclude the confounding effect of BMI on conduction defects due to unavailability of uniformly identifying patient height.

Several studies have demonstrated increased cardiovascular morbidity and mortality in NAFLD patients [25-26]. It is plausible that healthcare providers should treat patients with NAFLD for various cardiovascular risk factors in order to reduce their cardiovascular morbidity and mortality. Lifestyle modification, including weight loss, dietary modifications, and physical activity are the first line treatment for NAFLD and were shown to improve outcomes in patients with NAFLD [27-28]. More recently, thiazolidinediones (pioglitazone and rosiglitazone) and liraglutide were recommended for the management of NAFLD [29-30].

\section{Conclusions}

The role of NAFLD as a novel risk factor for cardiac disease has been extensively researched in the last decade. Previous studies have shown an association between NAFLD and structural and metabolic cardiac changes. Still, the data is relatively scarce for the association between NAFLD and arrhythmogenic cardiac abnormalities. Our study suggests that NAFLD is associated with conduction defects. Larger studies are needed to find out the causal relationship as well as the pathophysiological pathways that connect NAFLD and arrhythmogenic abnormalities of the heart.

\section{Additional Information \\ Disclosures}

Human subjects: All authors have confirmed that this study did not involve human participants or tissue. Animal subjects: All authors have confirmed that this study did not involve animal subjects or tissue. Conflicts of interest: In compliance with the ICMJE uniform disclosure form, all authors declare the following: Payment/services info: All authors have declared that no financial support was received from any organization for the submitted work. Financial relationships: All authors have declared that they have no financial relationships at present or within the previous three years with any organizations that might have an interest in the submitted work. Other relationships: All authors have declared that there are no other relationships or activities that could appear to have influenced the submitted work.

\section{Acknowledgements}

The authors thank Omer Zuberi, MD, First Coast Cardiovascular Institute for reviewing the manuscript.

\section{References}

1. Younossi ZM, Koenig AB, Abdelatif D, et al.: Global epidemiology of nonalcoholic fatty liver disease-Meta-analytic assessment of prevalence, incidence, and outcomes. Hepatology. 2016, 64:73-84. 10.1002/hep.28431

2. Chalasani N, Younossi Z, Lavine JE, et al.: The diagnosis and management of non-alcoholic fatty liver disease: practice guideline by the American Association for the Study of Liver Diseases, American College of Gastroenterology, and the American Gastroenterological Association. Am J Gastroenterol. 2012, 107:811-826. 10.1038/ajg.2012.128

3. Browning JD, Szczepaniak LS, Dobbins R, et al.: Prevalence of hepatic steatosis in an urban population in the United States: impact of ethnicity. Hepatology. 2004, 40:1387-1395. 10.1002/hep.20466

4. Markus MR, Meffert PJ, Baumeister SE, et al.: Association between hepatic steatosis and serum liver enzyme levels with atrial fibrillation in the general population: the Study of Health in Pomerania (SHIP). Atherosclerosis. 2016, 245:123-31. 
5. Nascimbeni F, Pais R, Bellentani S, et al.: From NAFLD in clinical practice to answers from guidelines. J Hepatol. 2013, 59:859-871. 10.1016/j.jhep.2013.05.044

6. Lonardo A, Bellentani S, Argo CK, et al.: Epidemiological modifiers of non-alcoholic fatty liver disease: focus on high-risk groups. Dig Liver Dis. 2015, 47:997-1006.

10.1016/j.dld.2015.08.004

7. Byrne CD, Targher G: NAFLD: a multisystem disease . J Hepatol. 2015, 62:47-64. 10.1016/j.jhep.2014.12.012

8. Armstrong MJ, Adams LA, Canbay A, et al.: Extra-hepatic complications of nonalcoholic fatty liver disease. Hepatology. 2014, 59:1174-1197. 10.1002/hep.26717

9. M.R. Markus, S.E. Baumeister, J. Stritzke: Hepatic steatosis is associated with aortic valve sclerosis in the general population. Arterioscler Thromb Vasc Biol. 2013, 33:1690-1695. 10.1161/ATVBAHA.112.300556

10. İşcen S: RBBB is associated with an increased risk of NAFLD in young healthy individuals . Int J Cardiol. 2013, 168:4056-4057. 10.1016/j.ijcard.2013.07.035

11. Mirbagheri SA, Rashidi A, Abdi S, et al.: Liver: an alarm for the heart? Liver Int. 2007, 27:891894. 10.1111/j.1478-3231.2007.01531.x

12. Açikel M, Sunay S, Koplay M, et al.: Evaluation of ultrasonographic fatty liver and severity of coronary atherosclerosis, and obesity in patients undergoing coronary angiography. Anatol J Cardiol. 2009, 9:273-9.

13. Wong VW, Wong GL, Yip GW: Coronary artery disease and cardiovascular outcomes in patients with non-alcoholic fatty liver disease. Gut. 2011, 60:1721-7.

10.1136/gut.2011.242016

14. Rijzewijk LJ, van der Meer RW, Smit JW, et al.: Myocardial steatosis is an independent predictor of diastolic dysfunction in type 2 diabetes mellitus. J Am Coll Cardiol. 2008, 52:1793-1799. 10.1016/j.jacc.2008.07.062

15. Thanassoulis G, Massaro JM, O'Donnell CJ, et al.: Pericardial fat is associated with prevalent atrial fibrillation: the Framingham Heart Study. Circ Arrhythm Electrophysiol. 2010, 3:345350. 10.1161/CIRCEP.109.912055

16. Lin Y-K, Chen YC, Chen J-H, et al.: Adipocytes modulate the electrophysiology of atrial myocytes: implications in obesity-induced atrial fibrillation. Basic Res Cardiol. 2012, 107:111. 10.1007/s00395-012-0293-1

17. Anstee QM, Targher G, Day CP: Progression of NAFLD to diabetes mellitus, cardiovascular disease or cirrhosis. Nat Rev Gastroenterol Hepatol. 2013, 10:330-344. 10.1038/nrgastro.2013.41

18. Targher G, Byrne CD: Nonalcoholic fatty liver disease: a novel cardiometabolic risk factor for type 2 diabetes and its complications. J Clin Endocrinol Metab. 2013, 98:483-495.

10.1210/jc.2012-3093

19. Chung MK, Martin DO, Sprecher D, et al.: C-reactive protein elevation in patients with atrial arrhythmias. Circulation. 2001, 104:2886-2891. 10.1161/hc4901.101760

20. Aviles RJ, Martin DO, Apperson-Hansen C, et al.: Inflammation as a risk factor for atrial fibrillation. Circulation. 2003, 108:3006-3010. 10.1161/01.CIR.0000103131.70301.4F

21. Guo Y, Lip GYH, Apostolakis S: Inflammation in atrial fibrillation. J Am Coll Cardiol. 2012, 60:2263-2270. 10.1016/j.jacc.2012.04.063

22. Castera L, Vilgrain V, Angulo P: Noninvasive evaluation of NAFLD. Nat Rev Gastroenterol Hepatol. 2013, 10:666-675. 10.1038/nrgastro.2013.175

23. Park SH, Kim PN, Kim KW, et al.: Macrovesicular hepatic steatosis in living liver donors: use of CT for quantitative and qualitative assessment. Radiology. 2006, 239:105-12. 10.1148/radiol.2391050361

24. Festi D, Schiumerini R, Marzi L, et al.: Review article: the diagnosis of non-alcoholic fatty liver disease -- availability and accuracy of non-invasive methods. Aliment Pharmacol Ther. 2013, 37:392-400. 10.1111/apt.12186

25. Younossi, Zobair M, Otgonsuren M, et al.: In patients with non-alcoholic fatty liver disease, metabolically abnormal individuals are at a higher risk for mortality while metabolically normal individuals are not. Metabolism. 2013, 62:352-360. 10.1016/j.metabol.2012.08.005

26. Rafiq N, Bai C, Fang Y, et al.: Long-term follow-up of patients with nonalcoholic fatty liver . Clin Gastroenterol Hepatol. 2009, 7:234-238. 10.1016/j.cgh.2008.11.005

27. Ratziu V, Bellentani S, Cortez-Pinto H, et al.: A position statement on NAFLD/NASH based on 


\section{Cureus}

the EASL 2009 special conference. J Hepatol. 2010, 53:372-384. 10.1016/j.jhep.2010.04.008

28. Loria P, Adinolfi LE, Bellentani S, et al.: Practice guidelines for the diagnosis and management of nonalcoholic fatty liver disease. Dig Liver Dis. 2010, 42:272-282. 10.1016/j.dld.2010.01.021

29. Belfort R, Harrison SA, Brown K, et al.: A placebo-controlled trial of pioglitazone in subjects with nonalcoholic steatohepatitis. N Engl J Med. 2006, 355:2297-2307.

10.1056/NEJMoa060326

30. Armstrong MJ, Gaunt P, Aithal GP, et al.: Liraglutide safety and efficacy in patients with nonalcoholic steatohepatitis (LEAN): a multicentre, double-blind, randomised, placebo-controlled phase 2 study. The Lancet. 2016, 387:679-90. 10.1016/S0140-6736(15)00803-X 